\title{
Eficiência de mercado: evidências empíricas para os preços spot e futuro de boi gordo
}

\author{
Waldemiro Alcântara da Silva Neto ${ }^{1}$ \\ Gilberto Joaquim Fraga ${ }^{2}$ \\ Pedro Valentin Marques ${ }^{3}$
}

\begin{abstract}
Resumo: O presente artigo aplica a hipótese de eficiência de mercado entre os preços spot do boi gordo de algumas relevantes praças no Brasil: Presidente Prudente, Goiânia e Campo Grande, e o preço futuro BM\&F. O procedimento adotado foi o de cointegração, para testar a eficiência de mercado sem implicar na ausência do prêmio de risco. Os resultados sugerem que não é possível rejeitar a hipótese de que o mercado é eficiente para as praças e não rejeitaram a hipótese da existência de prêmio de risco. Desta forma, o mercado futuro pode auxiliar no processo de descoberta de preços por parte dos agentes envolvidos.
\end{abstract}

Palavras-chave: eficiência de mercado; prêmio de risco; cointegração; pecuária de corte.

\section{Market efficiency: empirical evidence of spot and future prices of catlle}

\footnotetext{
Abstract: This article uses the hypothesis of efficiency market between spot prices of cattle to relevant places in Brazil: Presidente Prudente, Campo Grande and Goiania, and future price BM\&F. The co-integration procedure was adopted to test the efficiency of the market without implying the absence of risk premium. The results suggest that it is not possible to reject the hypothesis that the market is efficient in the places and they did not reject the hypothesis of the existence of risk premium. Thus, the futures market can be important in the process of discovery price by involved agents.

Professor Assistente FACE/UFG - wasneto@esalq.usp.br

Professor do Departamento de Economia - UEM- gjfraga@usp.br

Professor Titular do Departamento de Economia - USP/Esalq - pvmarque@ esalq.usp.br
} 
Keywords: market efficiency; risk premium; co-integration; beef cattle.

JEL: Q13; C51

\section{Introdução}

O setor agropecuário possui relevância consolidada na economia brasileira e, quanto ao seu perfil, verificam-se recorrentes possibilidades de riscos principalmente para os produtores, seja da formação de preços ou de acontecimentos inerentes à própria atividade, como secas, excesso de chuvas, pragas entre outros.

No período recente a eficiência do mercado de commodities agrícolas negociadas na BM\&F (Bolsa de Mercadorias e Futuros) vem sendo objeto de estudo de pesquisadores acadêmicos, conforme pode ser observado nos trabalhos de Amado e Carmona (2004), Duarte et al. (2007) e Abitante (2008). O objetivo desses autores tem sido o aumentar entendimento sobre o comportamento da descoberta de preços por parte dos agentes atuantes em determinado mercado, para, então, auxiliar os tomadores de decisões num cenário de risco.

Nesse contexto o presente trabalho tem como objetivo verificar a existência de uma relação de longo prazo entre o mercado físico e o mercado futuro da commodity boi gordo para algumas das principais regiões produtoras do Brasil. Atenção maior será dada na constatação da eficiência ou não do mercado, ou seja, verificar se o mercado futuro pode ser sugerido como um bom indicador para predizer o preço spot, portanto, auxiliando os agentes econômicos do setor no processo de descoberta do preço. Para alcançar o objetivo proposto serão utilizados procedimentos econométricos de séries temporais - testes de raiz unitária e vetores de cointegração.

Além desta introdução, este artigo trará ainda mais quatro partes. Na seguinte, um panorama sobre o setor e sua importância no Brasil. Posteriormente, uma breve revisão de literatura acerca da teoria da eficiência de mercado, dos modelos teóricos e da descrição dos dados utilizados e do modelo econométrico. Na quarta, a descrição dos principais resultados obtidos e por último, as considerações finais.

\section{Relevância da pecuária de corte no cenário nacional e inserção no internacional}

Nos últimos anos, o agronegócio tem se revelado fundamental para a economia brasileira. Seu PIB alcançou $\mathrm{R} \$ 611,80$ bilhões em 2007, com 
um crescimento de 7,89\% em relação ao ano anterior (CONFEDERAÇÃO DA AGRICULTURA E PECUÁRIA DO BRASIL 2008). No mesmo período, o crescimento do PIB nacional foi de $5,4 \%$.

A pecuária e os setores a montante e a jusante que a ela se ligam são responsáveis por aproximadamente um terço do valor da produção do agronegócio brasileiro, e se mostra uma cadeia estratégica para os anseios de crescimento e sustentabilidade da economia. Segundo dados da CEPEA-USP - Centro de Estudos Avançadas em Economia Aplicada e CNA, o valor bruto da produção da pecuária em 2007 foi de $\mathrm{R} \$ 68,43$ bilhões, já o agronegócio da pecuária, neste mesmo ano obteve $\mathrm{R} \$ 212,47$ bilhões e com previsão de alcance de $\mathrm{R} \$$ 263,12 bilhões em 2008.

Um estudo realizado pelo MAPA (2008) revela uma crescente participação do Brasil nas exportações mundiais de carne bovina, chegando a mais de 30\% nos dias de hoje. De outro lado, a carne brasileira tem posição de destaque entre os produtos agropecuários exportados pelo Brasil, sendo um produto importante quando se busca a geração de superávits na balança comercial (Miranda 2001).

O aumento das exportações brasileiras deste produto ocorre de forma mais expressiva a partir de 2002 e as projeções de crescimento até 2018 são de $2,48 \%$ ao ano (MAPA 2008). O Brasil, como mostra a Tabela 1, tornou-se o maior exportador em 2003, e vem consolidando cada vez mais esta posição.

TABELA 1. EXPORTAÇÕES MUNDIAIS DE CARNE BOVINA (MIL TONELADAS)

\begin{tabular}{lccccccc}
\hline & 2002 & 2003 & 2004 & 2005 & 2006 & $2007^{*}$ & Var. (o6/o2) \\
\hline Brasil & 1006 & 1301 & 1854 & 2198 & 2200 & 2420 & $118,69 \%$ \\
Austrália & 1366 & 1264 & 1394 & 1413 & 1420 & 1495 & $3,95 \%$ \\
Índia $^{1}$ & 417 & 439 & 499 & 627 & 750 & 800 & $79,86 \%$ \\
N. Zelândia & 486 & 558 & 606 & 589 & 540 & 570 & $11,11 \%$ \\
EUA & 1110 & 1142 & 2009 & 317 & 523 & 680 & $-52,88 \%$ \\
\hline
\end{tabular}

FONTE: USDA; ${ }^{1}$ Inclui Búfalo; *Previsão; Elaboração CNA.

Para um país exportador de carnes é importante também possuir uma pauta elevada de produtos e não basta apenas ter um grande número de países compradores. $\mathrm{O}$ fato de se exportar para um grande número de destinos reduz o risco associado às grandes variações na demanda interna dos países compradores. Ainda sobre o estudo realizado pelo MAPA, SPA e IICA (2007) há um alerta de que os melhores clientes são aqueles que compram em grande quantidade e também numa pauta diversificada de itens. A tabela a seguir mostra a relação dos principais importadores de carne brasileira em 2005, com destaque ao Reino Unido quanto ao valor, e a Arábia Saudita no que diz respeito à diversificação na pauta. Quanto ao valor médio por mil toneladas em milhões de dólares, o destaque é a Suécia. 
TABELA 2. PRINCIPAIS DESTINOS DAS EXPORTAÇÕES DE CARNE BOVINA, 2005 .

\begin{tabular}{ccccc}
\hline País & Pauta & $\begin{array}{c}\text { Valor (US\$ } \\
\text { milhóes) }\end{array}$ & $\begin{array}{c}\text { Quantidade } \\
\text { (mil } \\
\text { toneladas) }\end{array}$ & $\begin{array}{c}\text { Valor } \\
\text { médio (US\$ } \\
\text { milhóes/mil } \\
\text { toneladas)* }\end{array}$ \\
\hline Reino Unido & 4 & 249,70 & 99,40 & 2,51 \\
Rússia & 6 & 242,60 & 158,30 & 1,53 \\
Holanda & 6 & 229,90 & 54,50 & 4,22 \\
Chile & 4 & 199,90 & 105,10 & 1,90 \\
Estados Unidos & 5 & 197,60 & 55,60 & 3,55 \\
Itália & 4 & 157,40 & 48,60 & 3,24 \\
Irã & 1 & 102,10 & 63,60 & 1,61 \\
Alemanha & 6 & 95,30 & 23,80 & 4,00 \\
Hong Kong & 7 & 78,30 & 54,00 & 1,45 \\
Espanha & 6 & 78,00 & 20,40 & 3,82 \\
Arabia Saudita & 9 & 75,00 & 43,20 & 1,74 \\
Argélia & 3 & 61,70 & 38,80 & 1,59 \\
Israel & 7 & 44,30 & 24,80 & 1,79 \\
Suécia & 3 & 34,70 & 7,20 & 4,82
\end{tabular}

FONTE: MAPA (2007: 47) *Com a inclusão de informações adicionais pelos autores.

Os 14 principais países importadores da carne bovina brasileira em 2005 foram responsáveis por $80 \%$ do valor total exportado pelo Brasil. Trata-se de uma expressiva concentração diante do total de 106 países importadores. O produto de destaque é a carne bovina desossada e congelada, seguida pela fresca ou resfriada. Sendo assim, há um cenário otimista ao setor exportador, pois existe uma elevada gama de destinos à carne bovina brasileira, restando agora um trabalho intensivo em aumentar o volume de produto. Talvez o mais importante, a maior barreira já tenha sido vencida: o fato de o Brasil exportar para um grande número de países significa que já existem atendimentos sanitários estabelecidos com estes países importadores, o que significa maior facilidade e conhecimento desses mercados compradores. (MAPA et al. 2007: 47)

A quantidade efetiva de bovinos existentes no território brasileiro em 2006 ultrapassou 160 milhões de cabeças, considerando de forma conjunta a pecuária de corte e de leite (Figura 1). Do total de bovinos, os de corte representam 79,40\% naquele ano (ANUALPEC 2007). 


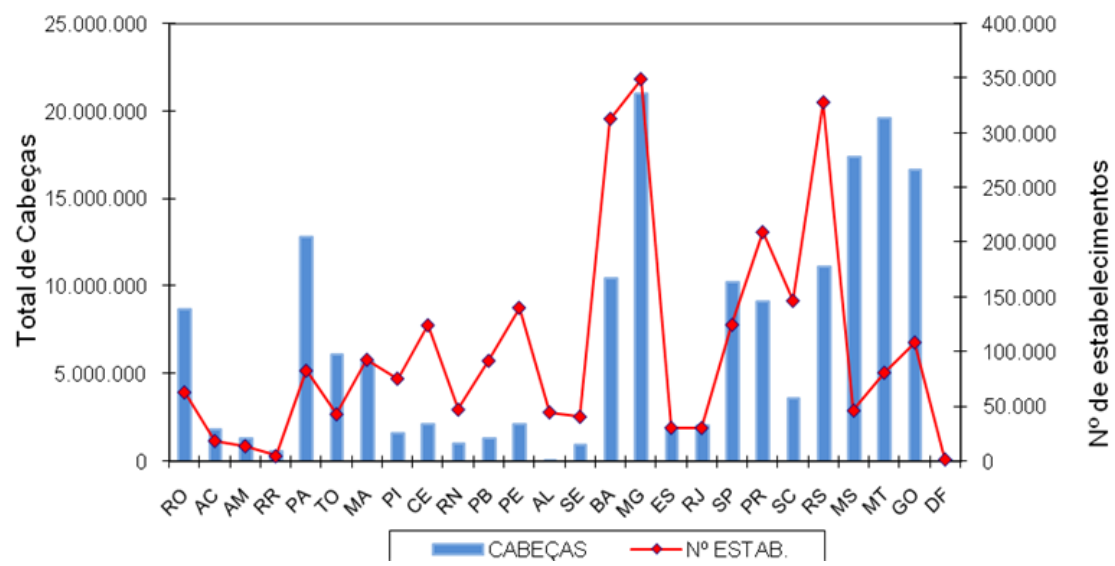

FIGURA 1. REBANHO BOVINO E NÚMERO DE ESTABELECIMENTOS POR UNIDADES DA FEDERAÇÃO EM 2006.

FONTE: IBGE (2008); ANUALPEC, 2007

Os maiores criadores encontram-se no eixo Centro-Sul, nos estados de Mato Grosso, Mato Grosso do Sul, Minas Gerais e Goiás, com participação conjunta de cerca de $45 \%$. O número de estabelecimentos nos estados da Região Centro-Oeste é pequeno comparativamente ao dos demais estados, o que mostra, considerando a grande produção do Centro-Oeste, que nesta região existem grandes latifúndios. Segundo dados preliminares do Censo Agropecuário de 2006, fornecidos pelo IBGE (2008), o número total de estabelecimentos envolvidos com a pecuária no Brasil é cerca de 2,65 milhões. O tamanho médio dos estabelecimentos no Centro-Oeste é de 275 hectares, contra 65 ha do resto do país.

Por outro lado, fica claro o significativo avanço brasileiro no processo de industrialização da carne bovina. No Brasil existem atualmente 10 grandes frigoríficos ${ }^{4}$ e, segundo o CEPEA - Centro de Estudos Avançadas em Economia Aplicada e CNA (2008), os 5 maiores frigoríficos são responsáveis por aproximadamente $38 \%$ da capacidade instalada de abate. A tecnologia de processamento avançou significativamente ao longo do tempo e hoje essas plantas são comparáveis, se não superiores, às americanas e europeias. Grupos brasileiros estão realizando investimentos em países como Estados Unidos, Austrália, Argentina e Uruguai. A figura 2 mostra a evolução dos abates de bovinos por estado. 


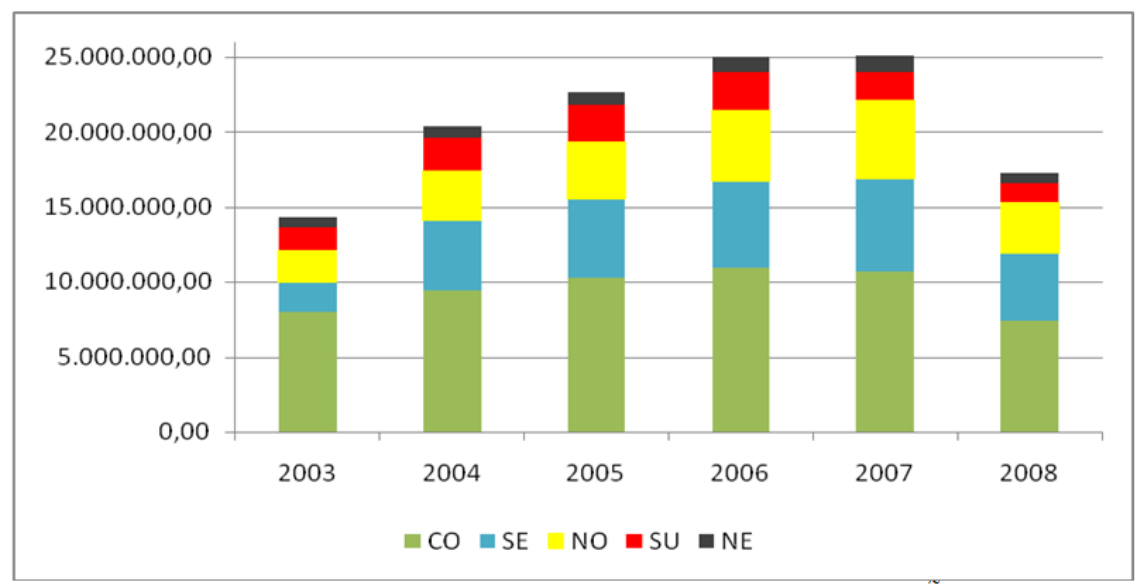

FIGURA 2. QUANTIDADE DE ABATE DE BOVINOS POR REGIÕES, DE 2003 A 2008*, EM MILHÕES DE CABEÇAS.

FONTE: MAPA (2008) elaborado pela ABIEC - Associação Brasileira das Indústrias Exportadoras de Carnes.

*Dados preliminares: até outubro.

A figura 2 mostra que a região Centro-Oeste é aquela que abate maior volume de bovinos, seguido pelo Sudeste e Norte. Fato esse que é corroborado pela maior presença de frigoríficos nesta região. No entanto, vale ressaltar que o estado de São Paulo é grande abatedor de bovinos, em 2007, foram abatidos cerca de 4,0 milhões de cabeças.

Dentre os principais destaques no setor estão: o avanço na tecnologia de processamento de carnes, crescimento nas exportações, consolidação na liderança mundial de oferta deste produto, melhoria nas condições do campo e boas expectativas de preços.

Diante disto, um fator fundamental para os agentes envolvidos é a questão de preços. Ou seja, os contratos entre ofertantes e demandantes de gado estão sendo fechados a todo o momento, e o desejo de que estes preços não sofram variações inversas ao desejado é muito grande. Desta forma justifica-se o objetivo deste artigo, que é o de testar a eficiência do mercado futuro de boi gordo no Brasil, através da análise de preços spot e futuro em algumas das principais praças de comercialização.

Caso esta desejada eficiência seja identificada, tanto os ofertantes (produtores/criadores de gado) e demandantes (frigoríficos/abatedouros), terão à sua disposição mais um mecanismo de predição de preços: a cotação do contrato futuro da BM\&F.

A possibilidade de negociar contratos futuros de boi gordo e de bezerro na 
Bolsa de Mercadorias e Futuros (BM\&F), que assegura menor risco de preço para a atividade, pode ser um fator determinante do maior nível de investimento e consequente desenvolvimento dessa cadeia.

A Tabela 3 trata da evolução do número de contratos futuros de boi gordo negociados na BM\&F desde 1998, observando-se que ele passou de quase 400 mil em 2006 para mais de 900 mil em 2007.

TABELA 3. NÚMERO DE CONTRATOS FUTUROS DE BOI GORDO NEGOCIADO NA BM\&F, CORRESPONDENTE NÚMERO DE ANIMAIS (BOIS) E \% DE ABATE DE 1998 A 2007.

\begin{tabular}{ccccccc}
\hline Ano & Contratos & $\begin{array}{c}\text { Variação } \\
(\%)\end{array}$ & $\begin{array}{c}\text { Correspondente } \\
\text { número de } \\
\text { animais }\end{array}$ & $\begin{array}{c}\text { \% do } \\
\text { abate }\end{array}$ & $\begin{array}{c}\text { Rebanho } \\
\text { (milhões) }\end{array}$ & $\begin{array}{r}\% \text { do } \\
\text { rebanho }\end{array}$ \\
\hline 1998 & 88.054 & - & 1.761 .080 & 6,11 & 155,3 & $1,13 \%$ \\
1999 & 123.442 & 40,19 & 2.468 .840 & 8,32 & 156,6 & $1,58 \%$ \\
2000 & 150.410 & 21,85 & 3.008 .200 & 9,58 & 160,5 & $1,87 \%$ \\
2001 & 92.365 & $-38,59$ & 1.847 .300 & 5,67 & 164,5 & $1,12 \%$ \\
2002 & 152.939 & 65,58 & 3.058 .780 & 8,75 & 169,2 & $1,81 \%$ \\
2003 & 113.473 & $-25,81$ & 2.269 .460 & 5,98 & 171,8 & $1,32 \%$ \\
2004 & 225.200 & 98,46 & 4.504 .000 & 10,43 & 172,9 & $2,61 \%$ \\
2005 & 311.459 & 38,30 & 6.229 .180 & 13,89 & 171,4 & $3,63 \%$ \\
2006 & 392.012 & 25,86 & 7.840 .240 & 16,59 & 165,0 & $4,75 \%$ \\
2007 & 934.422 & 138,37 & 18.688 .440 & - & 159,3 & $11,73 \%$ \\
\hline
\end{tabular}

FONTE: BM\&F; Anualpec 2007.

O avanço verificado na comercialização de contratos futuros de boi gordo junto à $\mathrm{BM} \& \mathrm{~F}$ entre 1998 e 2007 foi 961,19\% que representa 11,73\% do rebanho nacional, hedgiado. É um número expressivo, mas que ainda reflete uma grande possibilidade de crescimento.

\section{Eficiência de mercado: evidência empírica e modelo teórico}

\subsection{Eficiência de mercado: evidências empíricas a partir do Brasil}

No período recente a eficiência do mercado de commodities agrícolas negociadas na $\mathrm{BM} \& \mathrm{~F}$ vem sendo objeto de estudo de pesquisadores acadêmicos. $\mathrm{O}$ objetivo tem sido aumentar o entendimento do comportamento da descoberta de preços por parte dos agentes atuantes, para então, auxiliar os agentes tomadores de decisões num cenário de risco. 
Nessa linha teórica, Abitante (2008) buscou verificar a existência de uma relação de longo-prazo entre o mercado físico e o mercado futuro das commodities boi gordo e soja, no âmbito do Brasil. O autor buscou constatar se o mercado futuro pode fornecer informações que auxiliem os produtores no processo de descoberta de preço, e também, analisar a eficiência com que o mercado futuro pode predizer o preço spot. Para o boi gordo, o mercado futuro considerado é referente ao contrato (janeiro a novembro de 2005) da BM\&F, e no caso da soja foram analisados os contratos futuros (de março a setembro de 2005) de soja da BM\&F e da Chicago Board of Trade (CBOT). Para o mercado de boi gordo, foram encontradas evidências de cointegração entre os preços spot e os contratos com vencimento em março, abril, setembro, outubro e novembro de 2005. No caso do contrato de soja da BM\&F, a cointegração foi detectada nos vencimentos de abril, maio e novembro/2005. Já o de soja da CBOT apresentou cointegração nos vencimentos de janeiro, março, setembro e novembro de 2005. Quanto aos resultados encontrados pelo autor, foi possível constatar que não existe relação de cointegração para todos os contratos - o que não permite concluir sobre a eficiência do mercado. No entanto, o trabalho não focou nas explicações desse fato. Por outro lado, quanto ao indicador de eficiência do contrato futuro de boi gordo, o autor afirma que este se mostrou elevado.

Amado e Carmona (2004), com o intuito de verificar a eficiência do mercado futuro de commodities agrícolas no Brasil, utilizaram contratos futuros de três produtos negociados na BM\&F (açúcar, café e milho) no período de 1995 a 2003. Para alcançar o objetivo proposto os autores utilizaram procedimentos de séries temporais e, feitas as estimativas foi possível confirmar que as séries eram integradas de ordem 1. Em função da não estacionariedade das séries, foram realizados testes de cointegração e gerados modelos de correção de erro. As evidências sugerem que o preço futuro de café é um estimador não viesado do preço à vista e, os mercados de milho e açúcar são eficientes no primeiro período.

Com o objetivo de testar a eficiência do mercado futuro da soja no Brasil, Duarte et al. (2007) utilizaram análise de cointegração para preços spot e futuro, utilizando o procedimento de Johansen. O período analisado foi entre 03 de janeiro de 2005 a 24 de dezembro de 2005, conforme os resultados obtidos as séries de preços presente e futuro da soja são não estacionárias e integradas de primeira ordem. O teste dos parâmetros do modelo confirmou a hipótese nula de eficiência do mercado. Portanto, os autores chegaram à conclusão de que o mercado futuro da soja no Brasil pode ser considerado eficiente no período estudado.

\subsection{Modelo teórico}

Esta seção apresenta o modelo teórico a ser aplicado pelo presente estudo, a exposição será alicerçada nos modelos apresentados por Beck (1994) e Kellard (2002). Inicialmente os autores formulam os pressupostos teóricos do que 
seria um mercado eficiente para commodities, este implica que o preço futuro corrente, $\mathrm{F}_{\mathrm{t}}$, de um contrato futuro de commodity vencendo em $\mathrm{t}+1$ deve ser igual a esperança do preço spot em $\mathrm{t}+1$, ou seja, $F_{t}=E\left(S_{t+1} / I_{t}\right)$. Essa teoria está baseada no princípio de que os preços da commodity além de refletirem todas as informações, também estão disponíveis publicamente. Se os agentes participantes do mercado puderem usar informações adicionais para prever $\mathrm{S}_{\mathrm{t}+1}$, então eles atuarão comprando ou vendendo contratos futuros sempre que $F_{t} \neq E\left(S_{t+1} / I_{t}\right)$ até o mercado se equalizar. Desta forma, eficiência implica que $F_{t}$ é o melhor previsor de $S_{t+1}$, e também, $F_{t}$ deverá incorporar toda informação relevante disponível além do preço spot passado e preços futuros.

Para testar essa hipótese, Beck (1994) apresenta inicialmente a equação (1) a seguir. No entanto, variações dessa equação podem ser utilizadas para verificar eficiência.

$$
S_{t+1}=c_{0}+c_{1} F_{t}+u_{t+1}
$$

Sendo assim, o preço spot esperado é igual ao preço futuro corrente mais um componente de erro $(\mathrm{u})$ e, assumindo um cenário de expectativas racionais. A hipótese nula $\left(\mathrm{H}_{\mathrm{o}}\right)$ é $\mathrm{c}_{\mathrm{o}}=\mathrm{O}$ e $\mathrm{c}_{1}=1$.

A hipótese de que o preço futuro é um previsor não viesado do preço spot, é uma hipótese conjunta de que o mercado é eficiente e não há prêmio de risco ${ }^{5}$. Trabalhos empíricos mostram que a otimização intertemporal dos produtores avessos ao risco implicaria que o prêmio de risco criado pelas respectivas demandas de hedging causaria $\mathrm{c}_{\mathrm{o}} \neq \mathrm{O}$ e $\mathrm{c}_{1} \neq 1$ mesmo nos mercados eficientes (Beck 1994). Assim, é possível verificar através da técnica de cointegração que um mercado pode ser eficiente e talvez registrar a existência de prêmio de risco.

Para afirmar se as séries de preços spot e futuros são cointegradas, é necessário constatar se $u_{t+1}=S_{t+1}-c_{0}-c_{1} F_{t}$ é uma série estacionária. Num segundo momento, pode verificar a presença do prêmio de risco desde que, $\mathrm{c}_{\mathrm{o}}$ poderia não ser zero e c não ser necessariamente igual a um. Também, vale ressaltar que pode existir correlação serial nesse modelo, o que implicaria na dependência do preço passado do próprio spot. No entanto, isso violaria a eficiência do mercado.

Série cointegrada pode ser reescrita na especificação do modelo de correção do erro (MCE), conforme a equação (2) a seguir.

$$
\Delta S_{t+1}=a-\rho u_{t}+b \Delta F_{t}+\sum_{i=1}^{m} \beta_{i} \Delta S_{t+1-i}+\sum_{i=1}^{m} \gamma_{i} \Delta F_{t-i}
$$

onde $\rho>0$ porque mudanças nos preços a spot são respostas a desvios da

5 Beck (1994) admite que os participantes do mercado sejam neutros ao risco, no entanto, admite que isso não seja defensável teoricamente nem plausível empiricamente. 
relação de equilíbrio de longo prazo. Mercado eficiente implica em restrições adicionais: $\rho=1, \rho c_{1}=b \neq 0$ e $\beta_{i}=\gamma_{i}=0$, o coeficiente $\boldsymbol{b}$ do preço futuro corrente é diferente de zero porque todas as novas informações sobre mudanças no preço futuro corrente são repassadas para o preço spot futuro. Os coeficientes dos lags são zero porque as informações sobre o passado das séries são incorporadas no preço futuro corrente.

Se as restrições não se mantêm, então, o passado dos preços spot e futuro são informações importantes para prever $\mathrm{S}_{\mathrm{t}+1}$. Portanto, nem todas as informações disponíveis refletem completamente no preço futuro corrente, $\mathrm{F}_{\mathrm{t}}$. Desta forma, Beck (1994) afirma que o mercado futuro é ineficiente. Se esse teste não impôs o pressuposto de que $c_{0}=0$ e $c_{1}=1$ permitem-se assim a presença do prêmio de risco.

\subsection{Dados}

Para a realização deste trabalho, foram utilizados dados diários dos preços de boi gordo de 02 de janeiro de 2007 à 28 de julho de 2008, totalizando 385 observações. No que tange as cotações de contrato futuro, foram coletados os preços, junto a BM\&F, referentes ao último vencimento dos contratos. Os preços do mercado físico foram disponibilizados pelo CEPEA (Centro de Estudos Avançados em Economia Aplicada). As localidades escolhidas foram: Presidente Prudente-SP (PP), Campo Grande-MS (CG) e Goiânia-GO (GO). A escolha destas localidades é justificada pela grande representatividade na criação e comercialização de boi gordo. No gráfico a seguir é possível visualizar todas as séries de preços deste trabalho no período de análise.

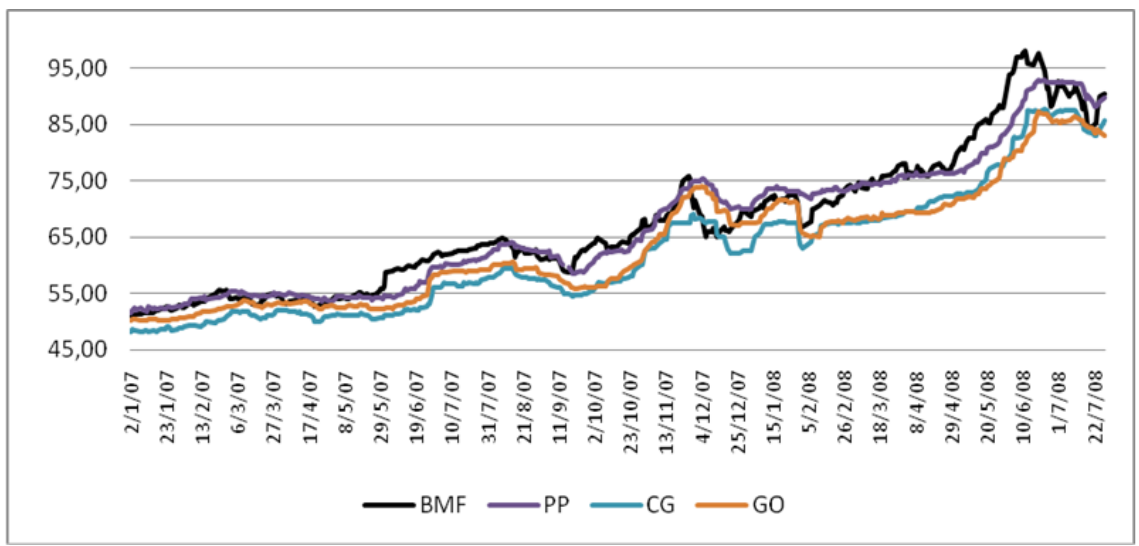

GRÁFICO 1. SÉRIE DE PREÇOS DIÁRIAS DE BOI GORDO: BM\&F, PRESIDENTE PRUDENTE-SP (PP), CAMPO GRANDE-MS (CG) E GOIÂNIA-GO (GO) DE o1 DE JANEIRO DE 2007 A 28 DE JULHO DE 2008, EM REAIS/@.

FONTE: CEPEA, BM\&F (2008) 


\subsection{Procedimentos econométricos}

\subsubsection{Teste de raiz unitária}

Em economia é comum as séries econômicas serem não estacionárias. Os preços não se comportam de maneira uniforme num intervalo de tempo, isto é, nem sempre os preços encontram-se num equilíbrio constante. Assim, tem-se a necessidade de se conhecer a estacionariedade das séries.

Portanto, com o objetivo de verificar a estacionariedade do índice geral de preços e dos alimentos, foi realizado o teste de raiz unitária de Dickey-Fuller Aumentado - ADF (1979). Seguindo os procedimentos apresentados por Enders (2004), o teste de raiz unitária ADF é utilizado para verificar a ordem de integração de uma série temporal $Y_{t}$, detectando ou não a existência de raiz unitária através dos seguintes modelos de regressão:

$$
\begin{aligned}
& \Delta Y_{t}=\alpha+\gamma+\rho Y_{t-1}+\sum \theta_{i} \Delta Y_{t-i}+\varepsilon_{t} \\
& \Delta Y_{t}=\alpha+\rho Y_{t-1}+\sum \theta_{i} \Delta Y_{t-i}+\varepsilon_{t} \\
& \Delta Y_{t}=\rho Y_{t-1}+\sum \theta_{i} \Delta Y_{t-i}+\varepsilon_{t}
\end{aligned}
$$

As estatísticas $\tau_{\tau}, \tau_{\beta \tau}, \tau_{\mu}$ e $\tau_{\alpha \mu}$, fornecidas por Mackinnon (1994), são todas usadas para testar a hipótese nula, $\rho=0$. É importante notar que a série pode ser estacionária com uma tendência determinista como na equação (03), ou sem tendência, mas, como drift como apresenta a equação (04).

Quanto à quantidade de lag(s) a ser utilizada, utilizam-se os critérios de Akaike (AIC), Schwarz (SBIC) e Hannan-Quinn (HQIC), para determinar o número $\mathrm{m}$ de defasagens autorregressivas a se considerar no teste de raiz unitária e em outros procedimentos econométricos propostos no estudo.

No caso das séries utilizadas não serem I(o) e serem integradas $\mathrm{I}(\mathrm{d})$ de mesma ordem, é necessário testar a cointegração entre elas. O conceito de cointegração está relacionado à existência de relação de longo prazo entre as variáveis. Para observar o comportamento da possível relação de longo-prazo entre as variáveis utilizam-se os procedimentos econométricos apresentados na próxima seção.

\subsubsection{Cointegração: apresentação teórica}

Muitas séries econômicas não são estacionárias em nível, mas possuem uma relação de equilíbrio de longo prazo. E, uma combinação linear dessas séries gera um desvio (erro) do equilíbrio de longo prazo que é estacionário. Essas séries estacionárias na primeira diferença também são conhecidas como 
séries integradas de ordem 1 ou I(1), e o termo de erro é I(o). Para constatar a presença (a quantidade) de vetores cointegrantes no Vetor de Correção do Erro (VEC) é utilizado o procedimento de Johansen (1988), que se baseia nos valores das estatísticas do traço $-\lambda_{\text {traço }}$, e a estatística do máximo autovalor $-\lambda_{\max }$, obtidos pelo método de verossimilhança (LR). Conforme Enders (2004), o VEC, em sua forma geral, para incluir termos deterministas, pode ser representado da seguinte forma:

$$
\Delta y_{t}=\alpha \beta^{\prime} y_{t-p}+\sum_{t=1}^{p-1} \Gamma_{i} \Delta y_{t-i}+\mu+\delta t+\varepsilon_{t}
$$

onde $y$ é um vetor $(k \times 1)$ de variáveis $\mathrm{I}(1)$, a e $\beta$ matriz de parâmetros $(k \times x)$ dos coeficientes de ajustamento e vetores cointegrantes respectivamente com rank $\mathrm{r}<\mathrm{k}, \Gamma_{1}, \ldots, \Gamma_{p-1}=\Gamma_{i}=-\left(\mathrm{I}-A_{1}-\ldots-A_{i}\right)$ é matriz $(k \mathrm{x} k)$ de parâmetros e, $\varepsilon_{\mathrm{t}}$ é um vetor $(k \times 1)$ de erros normalmente distribuídos e serialmente não correlacionados.

O número de vetores cointegrantes distintos pode ser obtido por meio da decomposição espectral da matriz $\alpha \beta^{\prime}=\Pi$, checando a significância de suas raízes características. Tomando as $n$ raízes características da matriz $\Pi$, $\lambda_{1}>\lambda_{2} \ldots .>\lambda_{n}$, são propostos então dois testes de razão de verossimilhança $(L R)$ com base nos autovalores estimados $\hat{\lambda}_{i}$. O primeiro produz a estatística do traço, como apresenta equação (05):

$$
\lambda_{\text {trace }}(r)=-T \sum_{i=r+1}^{k} \ln \left(1-\hat{\lambda}_{i}\right)
$$

sendo que a hipótese nula é o número de vetores cointegrantes e é igual a r, contra a hipótese alternativa de r menor que o número de vetores cointegrantes. O segundo teste produz a estatística do máximo autovalor, conforme a equação (08):

$$
\lambda_{\text {max }}(r, r+1)=-T \ln \left(1-\hat{\lambda}_{r+1}\right)
$$

Na equação acima a hipótese nula é de que existem r relações de cointegração, contra a hipótese alternativa de existência $r+1$ relações de cointegração. Se for constatado vetor de cointegração entre variáveis quaisquer relacionado a um modelo de transmissão, eles devem incluir um termo de correção de erro representado pelo resíduo da equação estimada. Por fim, feita essa breve apresentação dos procedimentos econométricos a serem utilizados, na próxima seção serão feitas as estimativas e apresentados os resultados encontrados.

\section{Resultados e discussões}

Inicialmente, e diante do objetivo de verificar o número de defasagens necessárias para eliminar a autocorrelação nos resíduos e nos demais procedimentos aplicados, seguem na tabela 4 os resultados do critério de informação. Para a escolha do número de defasagens (lag), foram adotados três critérios: Akaike information criterion (AIC); Hannan-Quinn information criterion (HQIC) e Scharz's Bayesian information criterion (SBIC). 
TABELA 4. RESULTADO DO TESTE DE SELEÇÃO DE DEFASAGEM

\begin{tabular}{|c|c|c|c|c|}
\hline Variável & $\begin{array}{l}\text { Defasagem } \\
\text { (lag) }\end{array}$ & AIC & $H Q I C$ & $S B I C$ \\
\hline \multirow[t]{3}{*}{ BM\&F } & 1 & 2.47659 & 2.48907 & 2.48376 \\
\hline & 2 & 2.42121" & $2.43353^{*}$ & 2.45226* \\
\hline & 3 & 2.42598 & 2.44241 & 2.46738 \\
\hline \multirow{4}{*}{ GO } & 1 & 1.17658 & 1.18479 & 1.19728 \\
\hline & 2 & 0.932368 & 0.944686 & 0.963414 \\
\hline & 3 & $0.898614 *$ & $0.915038^{*}$ & $0.940009^{*}$ \\
\hline & 4 & 0.903357 & 0.923887 & 0.9551 \\
\hline \multirow{4}{*}{ CG } & 1 & 1.35399 & 1.3622 & 1.37469 \\
\hline & 2 & 1.24684 & 1.25916 & 1.27789 \\
\hline & 3 & $1.23252^{*}$ & $1.24895^{*}$ & $1.27392^{*}$ \\
\hline & 4 & 1.2365 & 1.25703 & 1.28824 \\
\hline \multirow{4}{*}{ PP } & 1 & 1.0163 & 1.02451 & 1.037 \\
\hline & 2 & 0.988153 & 1.00047 & 1.0192 \\
\hline & 3 & 0.902753 & 0.919177 & 0.944148 \\
\hline & 4 & $0.860407^{*}$ & $0.880936^{*}$ & $0.912149^{*}$ \\
\hline
\end{tabular}

FONTE: Elaboração dos autores com dados do trabalho.

*) indica o número de defasagens a ser utilizado seguindo os critérios;

Para os preços futuros BM\&F de boi gordo, o número de defasagens para todos os critérios apresentados foi igual a três. Os de físico à vista em Goiânia (GO) e Campo Grande (CG) também apresentaram defasagem igual ao da BM\&F. Já Presidente Prudente (PP) revelou, segundo os critérios de informação, quatro lags.

Os resultados dos testes de raiz unitária para as séries de preços estão resumidos na tabela 5. O procedimento adequado segundo Enders (2004) exige partir de um modelo geral em direção ao específico, conforme descrito na seção 4.4.1 pelas equações (3), (4) e (5). 
TABELA 5. RESULTADO DO TESTE DE RAIZ UNITÁRIA DICKEYFULLER PARA AS VARIÁVEIS B\&MF, GO, PP, CG

\begin{tabular}{|c|c|c|c|c|}
\hline & Esta & icas $-A D F 1$ & $\begin{array}{c}\text { Ordem de } \\
\text { integraçãoo }\end{array}$ & lag \\
\hline \multicolumn{5}{|c|}{ Variáveis em nível } \\
\hline Variável & & $\tau_{\beta \tau}$ & $\mathrm{I}(1)$ & \\
\hline$B M \& F$ & -2.611 & 2.68 & $\mathrm{I}(1)$ & (2) \\
\hline GO & -2.466 & 2.51 & $\mathrm{I}(1)$ & (3) \\
\hline$C G$ & -1.865 & 2.18 & $\mathrm{I}(1)$ & $(3)$ \\
\hline$P P$ & -2.302 & 2.50 & $\mathrm{I}(1)$ & (4) \\
\hline \multicolumn{5}{|c|}{$\tau_{\beta \tau}:(\alpha, \beta, \rho)=(0,0,1) \mathrm{em} \mathrm{Y}_{t}=\alpha+\beta_{t}+\rho Y_{\mathrm{t}-1}+e_{\mathrm{t}}$} \\
\hline \multicolumn{5}{|c|}{ Variáveis em primeira diferença } \\
\hline$\triangle B M \& F$ & & 4.76 & & (2) \\
\hline$\Delta G O$ & & 7.59 & & (3) \\
\hline$\Delta C G$ & & 5.57 & & (3) \\
\hline$\triangle P P$ & & 2.35 & & (4) \\
\hline
\end{tabular}

FONTE: Elaboração dos autores com dados do trabalho.

Notas: ${ }^{1}$ ) Teste de Dickey-Fuller Aumentado.

Estatísticas (5\%): $\tau_{\tau}: 3,43 ; \tau_{\beta \tau}: 2,79 ; \tau_{\mu}: 2,88 ; \tau_{\alpha \mu}: 2,53$

O teste para a série de preços BM\&F, para o modelo geral descrito na equação (3), revela um valor calculado da estatística $\tau_{\tau}$ igual a 2,611, que é maior que o tabelado $\left(\tau_{\tau}: 3,43\right)$. Sendo assim, não é possível rejeitar a hipótese de presença de raiz unitária. Segue-se o mesmo resultado para o valor calculado da estatística $\tau_{\beta \tau}$ (existência de tendência), o valor tabelado para a estatística foi maior que o calculado. Logo, a série de preços do contrato futuro indicado pela $\mathrm{BM} \& \mathrm{~F}$ é não estacionária e possui um termo de tendência determinista.

Esta mesma sistemática, aplicada aos demais preços analisados, revela também que todas possuem raiz unitária e tendência determinista. Os valores

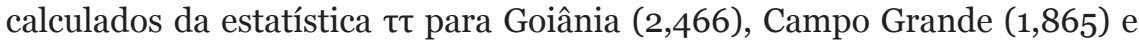
Presidente Prudente (2,302) são todos menores que o tabelado $\left(\tau_{\tau}: 3,43\right)$. A existência da tendência é indicada pela estatística $\tau_{\beta \tau}$, onde todos os valores calculados foram menores que o crítico ao nível de significância de 5\%, conforme reportado na tabela 4 .

Todas as séries foram testadas em primeira diferença, ainda conforme a tabela 4, e todas são estacionárias em torno de um termo de tendência. Portanto, as demais séries de preços analisadas são estacionárias em diferença, ou seja, integradas de ordem um (I(1)). Urso (2007), também encontrou resultados semelhantes acerca dos testes de raiz unitária, para dados semanais de setembro de 2000 a dezembro de 2006.

Dados que as séries não são estacionarias em nível, o próximo passo é realizar o teste de cointegração para os preços spot e futuro de boi gordo, sendo detalhados na tabela 6 . A coluna " $H_{o}=r$ " indica a hipótese que está sendo testada (a existência de zero vetores de cointegração ou a presença de um vetor). A 
decisão a ser tomada implica aceitar ou rejeitar que o número de vetores de cointegração seja igual a r. Ao rejeitar $H_{o}$ e aceitar $H_{1}$, indica a presença de vetor(es) de cointegração.

Na tabela 6, seguem-se os resultados da estatística de Johansen e pode-se constatar a existência de vetores de cointegração ${ }^{6}$, pois $H_{o}=1$ é rejeitado, logo não é possível rejeitar que $H_{1}>0$, onde o valor da estatística $\lambda_{\text {Trace }}$ é maior que o valor crítico à $5 \%$ para todas as séries consideradas. Também este resultado foi confirmado pela estatística $\lambda_{\text {máximo }}$. Sendo assim, através destes testes é possível concluir que há cointegração entre preços das praças (PP, GO e CG) e o preço futuro BM\&F do boi gordo.

\begin{tabular}{|c|c|c|c|c|c|c|}
\hline \multirow{2}{*}{$\begin{array}{c}H_{o} \\
\operatorname{Rank}=r\end{array}$} & \multirow{2}{*}{$\begin{array}{c}H_{1} \\
\text { Rank } \\
>r\end{array}$} & \multirow{2}{*}{ Eigenvalue } & \multicolumn{2}{|c|}{$\lambda_{\text {Trace }}$} & \multicolumn{2}{|c|}{$\lambda_{\text {máximo }}$} \\
\hline & & & estatística & $\begin{array}{c}\text { Valor } \\
\text { crítico 5\% }\end{array}$ & estatística & $\begin{array}{c}\text { valor } \\
\text { crítico } 5 \%\end{array}$ \\
\hline \multicolumn{7}{|c|}{$\mathrm{BM} \& \mathrm{~F}=>\mathrm{PP}$} \\
\hline $\mathrm{O}$ & $\mathrm{O}$ & - & 20,657 & 15,41 & 19,903 & 14,07 \\
\hline 1 & 1 & 0,180 & $0,753^{*}$ & 3,76 & 0,753 & 3,76 \\
\hline 2 & 2 & 0,007 & - & - & - & - \\
\hline \multicolumn{7}{|c|}{$\mathrm{BM} \& \mathrm{~F}=>\mathrm{GO}$} \\
\hline $\mathrm{o}$ & $\mathrm{O}$ & - & 21,974 & 15,41 & 21,144 & 14,07 \\
\hline 1 & 1 & 0,191 & $0,830^{*}$ & 3,76 & 0,830 & 3,76 \\
\hline 2 & 2 & 0,008 & - & - & - & - \\
\hline \multicolumn{7}{|c|}{$\mathrm{BM} \& \mathrm{~F}=>\mathrm{CG}$} \\
\hline $\mathrm{O}$ & 0 & - & 24,836 & 15,41 & 24.061 & 14,07 \\
\hline 1 & 1 & 0,214 & $0,775^{*}$ & 3,76 & 0,775 & 3,76 \\
\hline 2 & 2 & 0,008 & - & - & - & - \\
\hline
\end{tabular}

FONTE: Elaboração dos autores com dados do trabalho - STATA.

Notas: *) Significativo em nível de $5 \%$.

Estes resultados estão em linha com a literatura empírica brasileira conforme mostram os resultados alcançados. Abitante (2008) identificou a presença de séries cointegradas, mesmo com a presença de um vetor de tendência determinista. No entanto, isto foi verificado apenas para alguns vencimentos (meses de abril e setembro de 2005).

A presença de cointegração, conforme os resultados expostos na tabela 6, sugere que os mercados são eficientes. Esse comportamento indica também uma relação de equilíbrio de longo-prazo entre os dois preços (spot e futuro), em que diferenças nas trajetórias dos mesmos seriam motivadas apenas por choques de curto-prazo. Neste trabalho, há existência de vetores de coin-

6 Cabe destacar, que embora os testes apresentados em conjunto na tabela 5 (as quatro séries de preços), também foi realizado para os pares (preço físico de cada praça e futuro BM\&F) e, também, encontrou-se um vetor de cointegração entre estes pares. 
tegração entre os três pares spot (PP, GO e CG) e o futuro (BM\&F). Sendo assim, os preços spot nas cidades mantêm uma relação de longo prazo com os preços futuros da BM\&F.

Prosseguindo na análise da eficiência, foi estimado o modelo de correção do erro (MCE) - equação 02, onde o objetivo é verificar se há ou não prêmio de risco. A seguir, a tabela 7 apresenta os resultados do modelo de correção do erro.

TABELA 7. MODELO DE CORREÇĀO DO ERRO (MCE)

\begin{tabular}{|c|c|c|}
\hline \multicolumn{3}{|c|}{ Equação (02): $\Delta S_{t+1}=\alpha-\rho u_{t}+b \Delta F_{t}+\sum_{i=1}^{m} \beta_{i} \Delta S_{t+1-i}+\sum_{i=1}^{m} \gamma_{i} \Delta F_{t-i}$} \\
\hline Variável & Modelo Estimado & $R^{2}$ \\
\hline$C G$ & $\begin{aligned} & \Delta C G_{t+1}= 0,164-1.617 u_{t}-0,187 \Delta B M F_{t}-0,484 \Delta B M F_{t-1} \\
&(0,24) \quad(-6,83)^{*} \quad(-0,63) \\
&(-1,75)^{* *}\end{aligned}$ & 0,49 \\
\hline$G O$ & $\begin{aligned} \Delta G O_{t+1}= & 0,093-1.132 u_{t}-0,140 \Delta B M F_{t}-0,494 \Delta B M F_{t-1} \\
& (0,14) \quad(-5,40)^{*} \quad(-0,50)(-1,83)^{* *}\end{aligned}$ & 0,43 \\
\hline$P P$ & 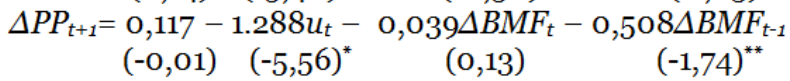 & 0,42 \\
\hline
\end{tabular}

Notas: números em parênteses são as estatísticas $\mathrm{z}$ (chi-quadrado) onde $\mathrm{H}_{\mathrm{o}}$ : $\alpha=b=\beta=\gamma=O$

$\left.{ }^{*}\right)$ significante a $1 \% ;{ }^{* *}$ ) significante a $10 \%$;

FONTE: Elaboração a partir dos dados do trabalho.

Estatisticamente, os resultados encontrados para o MCE indicam que não é possível rejeitar a hipótese de existência de prêmio de risco. Tendo em vista que os pressupostos teóricos foram violados conforme a tabela anterior, sendo os valores de $b$ e $y_{\mathrm{i}}$ diferentes dos parâmetros teoricamente esperados e, também, $B M F_{t-1}$ está influenciando o preço spot ${ }_{t+1}$, onde esperava-se que este fosse influenciado apenas por $B M F_{t}$. Desta forma, pode-se ver que o preço futuro é um previsor para o spot nas praças aqui consideradas, mas deve ser considerada a existência do prêmio de risco.

\section{Considerações finais}

O objetivo deste trabalho foi verificar a existência de uma relação de longo prazo entre os preços no mercado físico (spot) de algumas praças (Presidente Prudente, Goiânia e Campo Grande), e o mercado futuro da commodity boi gordo. Além disso, visou também constatar uma possível eficiência ou não do mercado, ou seja, verificar se o mercado futuro pode ser sugerido como um bom indicador para predizer o preço spot, auxiliando os agentes econômicos do setor no processo de descoberta do preço futuro.

A existência de cointegração em todas as séries analisadas sinaliza que o preço futuro pode auxiliar na descoberta do preço spot $\mathrm{t}+1$ por parte do pecuarista e dos demais agentes envolvidos neste mercado, conforme os pressupostos 
da teoria de eficiência de mercado.

Por fim, os resultados do trabalho sugerem que não é possível rejeitar a hipótese de que o mercado é eficiente. Quanto ao premio de risco, as estimativas mostraram estatisticamente que não foi possível rejeitar a sua existência. Como sugestão para os agentes atuantes nesse setor, é possível afirmar que os preços futuros são importantes para auxiliarem na previsão do preço spot, em particular nas praças analisadas, conforme os resultados encontrados.

\section{Referências}

ABITANTE, K. G. (2008). Co-integração entre os mercados spot e futuro: evidências dos mercados de boi gordo e soja. Revista de Economia e Sociologia Rural, 46 (1): $75-96$.

ALVES, J. S. \& DUARTE, G. S. \& LIMA, R. C. (2007). "Co-integração e Eficiência do Mercado Futuro da Soja no Brasil”. In XLV Congresso da Sociedade Brasileira de Economia e Sociologia Rural.

AMADO, C. F. P.; CARMONA, C. U. M. (2004). "Uma Análise da Eficiência dos Mercados Futuros Agrícolas Brasileiros”. In IV Encontro Brasileiro de Finanças, 2004, Rio de Janeiro: Coppead, UFRJ.

ANUÁRIO BRASILEIRO DA PECUÁRIA (ANUALPEC). (2008) São Paulo, FNP: Consultoria \& Comércio.

BECK, S. E. (1994). "Cointegration and market efficiency in commodity futures markets". Applied Economics, 26: 249-257.

BM\&F, Bolsa de Mercadorias e Futuro (2008). URL: http://www.bmf.com.br. Acesso em: setembro de 2008.

CENTRO DE ESTUDOS AVANÇADAS EM ECONOMIA APLICADA (CEPEA); CONFEDERAÇÃO NACIONAL DA AGRICULTURA E PECUÁRIA DO BRASIL (CNA). (2008). Ativos da Pecuária de Corte. Ano 2, Ed. 5, Abril de 2008.

CONFEDERAÇÃO NACIONAL DA AGRICULTURA E PECUÁRIA DO BRASIL (CNA). (2008) URL: http://www.cna.gov.br. Acesso em: 17 setembro de 2008.

ENDERS, W. (2002) Applied econometric time series. New York: John Wiley \& Sons. 466 p.

INSTITUTO BRASILEIRO DE GEOGRAFIA E ESTATÍSTICA - IBGE. (2008). URL: http://www.ibge.gov.br/estadosat/. Acesso em: o8 de setembro de 2008.

MACEDO, L. O. B. (2006) “Modernização da Pecuária de Corte Bovina no Brasil e a Importância do Crédito Rural”. Informações Econômicas, 36 (7):84-95.

MAPA, Ministério da Agricultura, Pecuária e Abastecimento. (2007). URL: http:// www.agricultura.gov.br/ . Acesso em: 17 de abril de 2008.

MARQUES, P. V \& MELLO, P. C. \& MARTINES FILHO, J. (2007). Mercados Futuros Agropecuários: exemplos e aplicações para o mercado brasileiro. Campus.

MCKENZIE, A. M. \& HOLT, M. (2002). "Market efficiency in agricultural futures Markets”. Applied Economics 34: 1519-1532. 
MINISTÉRIO DA AGRICULTURA, PECUÁRIA E ABASTECIMENTO (MAPA) \& SECRETARIA DE POLÍTICA AGRÍCOLA (SPA) \& INSTITUTO INTERAMERICANO DE COOPERAÇÃO PARA A AGRICULTURA (IICA). (2007). Cadeia Produtiva de Carne Bovina. Série Agronegócios, 8.

MIRANDA, S. H. G. (2001). Quantificação dos efeitos das barreiras não-tarifárias sobre as exportações brasileiras de carne bovina. São Paulo: Tese de Doutorado - Escola Superior de Agricultura Luiz de Queiroz, Universidade de São Paulo.

TOMEK, W. G. \& PETERSON, H. H (2001). "Risk management in agricultural markets: a review”. Journal of Futures Markets 21: 953-985.

URSO, F. S. P. (2007). A Cadeia Da Carne Bovina No Brasil: Uma Análise De Poder De Mercado E Teoria Da Informação. São Paulo: Tese de Doutorado - Fundação Getulio Vargas - Escola de Economia de São Paulo.

Recebido em: 14 de maio de 2009

Primeira resposta em: 08 de outubro de 2009

Aceite em: 08 de outubro de 2009 